\title{
BLOOD VESSELS SEGMENTATION METHOD FOR RETINAL FUNDUS IMAGES BASED ON ADAPTIVE PRINCIPAL CURVATURE AND IMAGE DERIVATIVE OPERATORS
}

\author{
Dang N. H. Thanh ${ }^{1}$, Dvoenko Sergey ${ }^{2}$, V. B. Surya Prasath ${ }^{3,4,5,6}$, Nguyen Hoang Hai ${ }^{7}$ \\ ${ }^{1}$ Department of Information Technology, Hue College of Industry, Vietnam - dnhthanh@hueic.edu.vn \\ ${ }^{2}$ Department of Information Security, Tula State University, Russia - dsd@tsu.tula.ru \\ ${ }^{3}$ Division of Biomedical Informatics, Cincinnati Children's Hospital Medical Center, USA \\ ${ }^{4}$ Department of Pediatrics, University of Cincinnati, OH USA \\ ${ }^{5}$ Department of Biomedical Informatics, College of Medicine, University of Cincinnati, USA \\ ${ }^{6}$ Department of Electrical Engineering and Computer Science, University of Cincinnati, USA - prasatsa@uc.edu \\ ${ }^{7}$ Department of Informatics, University of Education, University of Danang, Vietnam - hoanghai@ued.udn.vn
}

\section{Commission WG II/5, WG II/10}

KEY WORDS: Blood Vessels, Image Segmentation, Retinal Fundus Images, Principal Curvatures, Derivative Operator.

\begin{abstract}
:
Diabetes is a common disease in the modern life. According to WHO's data, in 2018, there were $8.3 \%$ of adult population had diabetes. Many countries over the world have spent a lot of finance, force to treat this disease. One of the most dangerous complications that diabetes can cause is the blood vessel lesion. It can happen on organs, limbs, eyes, etc. In this paper, we propose an adaptive principal curvature and three blood vessels segmentation methods for retinal fundus images based on the adaptive principal curvature and images derivatives: the central difference, the Sobel operator and the Prewitt operator. These methods are useful to assess the lesion level of blood vessels of eyes to let doctors specify the suitable treatment regimen. It also can be extended to apply for the blood vessels segmentation of other organs, other parts of a human body. In experiments, we handle proposed methods and compare their segmentation results based on a dataset - DRIVE. Segmentation quality assessments are computed on the Sorensen-Dice similarity, the Jaccard similarity and the contour matching score with the given ground truth that were segmented manually by a human.
\end{abstract}

\section{INTRODUCTION}

Computer-aided diagnosis for various diseases is a modern technology that plays a vital role to assist doctors in the interpretation of the medical images. The medical image processing techniques are helpful to detect early lesions inside human body. So, medical image processing in particular, and image processing in general are widely studied all over the world.

According to the WHO's data, there were $8.3 \%$ of adult population had diabetes. The diabetes is a common disease in the modern life that causes many lesions in the blood vessels of eyes, kidneys, heart, nerves, etc. The diabetic retinopathy is a primary cause of blindness (Patwari, et al., 2014). The early symptoms that can be visible on the retinal fundus image are lesions on blood vessels of retinal fundus of patients. So, the task of applying the image processing techniques to process the retinal fundus images are really necessary.

The blood vessels segmentation for the retinal fundus images plays very important role in the medical image processing. Unlike other medical segmentation tasks of organs, bone, brain, etc., the blood vessels are very small, and their intensity is very similar to intensity of other parts of retinal fundus images. So, the blood vessels segmentation problem is really a big challenge.

Because blood vessels segmentation is an important problem in medical image processing, it has attracted a lot of attention. There are many methods were developed, such as the Gabor filters with fractional derivatives and Expectation Maximization (Hugo, et al., 2018), supervised methods (Ngo \& Han, 2017), multiscale line detection (Aigerim \& Mahmud, 2015), scale space analysis, morphological processing (Moccia, et al., 2018), etc.

In this paper, we proposed three blood vessels segmentation methods for the retinal fundus images based on the adaptive principal curvature and the derivatives: the central difference, the Sobel and the Prewitt operators. The principal curvatures proved the potential to improve the line/edges contrast to get clearer (Hongli, et al., 2007). The principal curvatures are better than the gradient magnitude to improve the contrast of the lines/edges, because the gradient magnitude focuses on the borders, but the principal curvatures - on the sketch structure. So, the principal curvatures are more helpful for blood vessels segmentation and they are also helpful for segmenting buildings, roads of aerial/satellite images.

Otherwise, we also proposed a new principal curvature, that is called to be an adaptive principal curvature. This principal curvature is a combination of the maximum and the minimum principal curvatures. This combination is expected to be better than the maximum and the minimum principal curvatures.

In the experiment, we handle the proposed methods to segment the blood vessels of retinal fundus images of DRIVE dataset. The segmentation quality is assessed by the Sorensen-Dice metric, the Jaccard metric (Abdel \& Allan, 2015) and the contour matching score (Gabriela, et al., 2013). The segmentation quality is compared to the ground truth that is segmented manually. The ground truth is also given in the DRIVE dataset.

Our contribution focuses on applying the principal curvatures to improve blood vessel structures and proposing an adaptive 
principal curvature. Although the maximum principal curvature was used in several works of pattern recognition and computer vision, its application for the blood vessel segmentation problem is also a novelty of this work. However, we mainly focus on the adaptive principal curvature, because it enhances blood vessel structures better that we will show in the experiments. Otherwise, combination of some other image enhancement methods to improve segmentation quality is also a part of our contribution.

The rest of the paper is organized as follows. Section 2 presents the proposed blood vessels segmentation methods. Section 3 presents experimental results for blood vessels segmentation and the comparison. Finally, Section 4 is the conclusions.

\section{PROPOSED BLOOD VESSELS SEGMENTATION METHODS}

\subsection{Adaptive Principal Curvature}

Let us consider that $u(\boldsymbol{x})$ is an input image need to be segmented, where $\boldsymbol{x}=\left(x_{1}, x_{2}\right) \in \Omega \subset \mathbb{R}^{2}, \Omega$ is the image domain and $u(\boldsymbol{x}) \in \mathbb{R}$ (e.g. the input image is grayscale image). To segment the colour image, we can use separate colour channels of the RGB colour model (or other colour models, such as CIE Lab, CIE $\mathrm{XYZ}$ etc.), or simply convert the colour image to a grayscale. The consideration on grayscale images in $\mathbb{R}$ is also similar to on RGBcolourful images in $\mathbb{R}^{3}$.

We consider that the image $u(\boldsymbol{x})$ is a regular surface. Let $\boldsymbol{\eta}$ be a unit tangent vector of the surface $u$. Then, the normal curvature of the surface $u$ in the direction $\boldsymbol{\eta}$ is defined as:

$$
\kappa(\boldsymbol{\eta})=\mathcal{S}(\boldsymbol{\eta}) \cdot \boldsymbol{\eta}
$$

where $\mathcal{S}$ is a shape operator (e.g. the second fundamental tensor) as the tangential component of the rate of the change of the normal vector when moved along the curve of the surface tangent to the tangent vector.

Let us consider that $\lambda_{1}, \lambda_{2}, \ldots-$ values of the normal curvature $\kappa(\boldsymbol{\eta})$ at a given point on the regular surface. Then, the maximum and the minimum of the normal curvature (Gray, 1997) at a given point on a surface are called to be principal curvatures.

The maximum principal curvature $\lambda^{+}$and the minimum principal curvature $\lambda^{-}$can be computed by the Gaussian curvature $\mathcal{H}$ and the mean curvature $\mathcal{K}$ :

$$
\mathcal{H}=\operatorname{det}(\mathcal{S}(\boldsymbol{x})), \quad \mathcal{K}=1 / 2 \operatorname{tr}(\mathcal{S}(\boldsymbol{x})),
$$

where det is a matrix determinant, tr is a matrix trace (sum of elements of the main diagonal).

Let us consider that the shape operator at a given point is:

$$
\mathcal{S}=\left(\begin{array}{ll}
s_{11} & s_{12} \\
s_{21} & s_{22}
\end{array}\right), s_{12}=s_{21} .
$$

Then, the Gaussian curvature and the mean curvature are:

$$
\mathcal{H}=s_{11} s_{22}-s_{12}^{2}, \quad \mathcal{K}=1 / 2\left(s_{11}+s_{22}\right) .
$$

On the other hand, if $\lambda_{1}$ and $\lambda_{2}$ are eigenvalues of the shape operator, then we have:

$$
\operatorname{det}(\delta)=\lambda_{1} \lambda_{2}, \quad \operatorname{tr}(\delta)=\lambda_{1}+\lambda_{2} .
$$

So, $\lambda_{1}, \lambda_{2}$ are solutions of the equation:

$$
\lambda^{2}-2 \mathcal{K} \lambda+\mathcal{H}=0
$$

for the variable $\lambda \in \mathbb{R}$. Then, we have solutions:

$$
\lambda_{1}, \lambda_{2}=\mathcal{K} \pm \sqrt{\mathcal{K}^{2}-\mathcal{H}}
$$

Finally, we acquire values of the principal curvatures:

$$
\begin{aligned}
& \lambda^{+}=\max \left\{\lambda_{1}, \lambda_{2}\right\}=\mathcal{K}+\sqrt{\mathcal{K}^{2}-\mathcal{H}}, \\
& \lambda^{-}=\min \left\{\lambda_{1}, \lambda_{2}\right\}=\mathcal{K}-\sqrt{\mathcal{K}^{2}-\mathcal{H}} .
\end{aligned}
$$

The maximum principal curvature detects the dark lines/edges on the light background. The minimum principal curvature detects the light lines/edges on the dark background (Hongli, et al., 2007).

As we can see, the evaluation of Gaussian and mean curvatures reaches the definition of the shape operator $\mathcal{S}$. To define the shape operator concretely, we need to solve the Weingarten equations (Gray, 1997). To simplify the evaluation, we consider that the image is a surface, in that curvilinear structures (e.g. lines such as roads in aerial or satellite images or blood vessels in medical images) correspond to its ridges (high curvatures) and valleys (low curvatures). Then, the local shape of characteristics of the surface at a given pixel $\boldsymbol{x}=(i, j)$, where $i \in\{1,2, \ldots, m\}$, $j \in\{1,2, \ldots, n\}, m$ and $n$ are numbers of pixels by horizon and vertical, respectively, can be expressed by the Hessian:

$$
\mathbb{H}(\boldsymbol{x}, \sigma)=\left(\begin{array}{cc}
\frac{\partial^{2} w}{\partial x_{1}^{2}}(\boldsymbol{x}) & \frac{\partial^{2} w}{\partial x_{1} \partial x_{2}}(\boldsymbol{x}) \\
\frac{\partial^{2} w}{\partial x_{1} \partial x_{2}}(\boldsymbol{x}) & \frac{\partial^{2} w}{\partial x_{2}^{2}}(\boldsymbol{x})
\end{array}\right),
$$

where $\sigma$ is a standard deviation, and it plays the role of a spatial scale parameter of the Gaussian kernel for the low pass filters in a window with odd size:

$$
G_{\sigma}(\boldsymbol{y})=\frac{1}{2 \pi \sigma^{2}} \exp \left(-\frac{y_{1}^{2}+y_{2}^{2}}{2 \sigma^{2}}\right),
$$

where $\boldsymbol{y}=\left(y_{1}, y_{2}\right), y_{1}, y_{2} \in\{1,3,5, \ldots\}$, and $w=G_{\sigma}(\boldsymbol{y}) \star u$, where $\star$ denotes $2 \mathrm{D}$ convolution. Therefore, $w$ is a result of applying the 2D convolution of the Gaussian kernel with the standard deviation $\sigma$ to the input image $u$. In practice, the symmetric Gaussian kernel (i.e. $y_{1}=y_{2}$ ) is usually used.

Hence, the principal curvatures at a given pixel can be computed as eigenvalues of the Hessian at that pixel. The evaluation of the principal curvatures is handled on every pixel of the image. This means that the maximum and the minimum principal curvatures of the image $u$ are matrices that have the same size with the input image $u$.

Let us consider that $\lambda_{i j}^{(1)}, \lambda_{i j}^{(2)}$ are eigenvalues of the Hessian at every pixel $(i, j)$. Then, the maximum and the minimum principal curvatures are defined as:

$$
\begin{aligned}
& \lambda_{i j}^{+}=\max \left\{\lambda_{i j}^{(1)}, \lambda_{i j}^{(2)}\right\}, \\
& \lambda_{i j}^{-}=\min \left\{\lambda_{i j}^{(1)}, \lambda_{i j}^{(2)}\right\} .
\end{aligned}
$$

Let $\Lambda^{+}, \Lambda^{-}$be matrices of the maximum principal curvature and the minimum principal curvature, respectively. That means

$$
\Lambda^{+}=\left(\lambda_{i j}^{+}\right)_{m \times n}, \quad \Lambda^{-}=\left(\lambda_{i j}^{-}\right)_{m \times n} .
$$

We call the matrix

$$
\Lambda=\frac{3 \Lambda^{+}+\Lambda^{-}}{2}
$$

to be a matrix of the adaptive principal curvature.

The adaptive principal curvature is expected to improve blood vessels structure better than the maximum principal curvature and the gradient magnitude because the adaptive principal curvature combines both the maximum and minimum principal curvatures. Hence, contrast/brightness of blood vessels will be enhanced better. 


\subsection{Derivative Operators}

To evaluate the adaptive principal curvature of the image, we need to compute the second order derivatives. We consider three methods presented below.

\section{Derivatives by the central difference}

Derivatives by the central difference (Gonzalez \& Woods, 2008) can be computed as follows:

$$
\begin{aligned}
& \frac{\partial u}{\partial x_{1}}=\frac{u(i+1, j)-u(i-1, j)}{2}, \\
& \frac{\partial u}{\partial x_{2}}=\frac{u(i, j+1)-u(i, j-1)}{2},
\end{aligned}
$$

where $i, j$ are spatial steps by the image width and the image height, respectively.

By using the central difference, the derivative of specific row/column is evaluated by two neighbour rows/columns.

\section{The Sobel derivative operator}

Derivatives are evaluated by the Sobel operator (Gonzalez \& Woods, 2008) as follows:

$$
\begin{aligned}
\frac{\partial u}{\partial x_{1}} & =\left(\left(\begin{array}{l}
1 \\
2 \\
1
\end{array}\right)\left(\begin{array}{lll}
1 & 0 & -1
\end{array}\right)\right) \star u=\left(\begin{array}{ccc}
1 & 0 & -1 \\
2 & 0 & -2 \\
1 & 0 & -1
\end{array}\right) \star u, \\
\frac{\partial u}{\partial x_{2}} & =\left(\left(\begin{array}{l}
1 \\
2 \\
1
\end{array}\right)\left(\begin{array}{lll}
1 & 0 & -1
\end{array}\right)\right) \star u=\left(\begin{array}{ccc}
1 & 2 & 1 \\
0 & 0 & 0 \\
-1 & -2 & -1
\end{array}\right) \star u,
\end{aligned}
$$

where $T$ is the transposition. By using the Sobel operator, we can compute the directional derivatives by horizon and vertical at the same time. However, the Sobel operator uses three rows/columns to evaluate the derivative. It considers the weight of the specific row/column higher than the weights of two neighbouring rows/columns. Therefore, the Sobel operator preserves details of specific rows/columns better than the central difference. The Sobel operator is also used to detect the image edges.

\section{The Prewitt derivative operator}

Derivatives are evaluated by the Prewitt operator (Gonzalez \& Woods, 2008) as follows:

$$
\begin{aligned}
\frac{\partial u}{\partial x_{1}} & =\left(\left(\begin{array}{l}
1 \\
1 \\
1
\end{array}\right)\left(\begin{array}{lll}
1 & 0 & -1
\end{array}\right)\right) \star u=\left(\begin{array}{ccc}
1 & 0 & -1 \\
1 & 0 & -1 \\
1 & 0 & -1
\end{array}\right) \star u, \\
\frac{\partial u}{\partial x_{2}} & =\left(\left(\begin{array}{l}
1 \\
1 \\
1
\end{array}\right)\left(\begin{array}{lll}
1 & 0 & -1
\end{array}\right)\right) \star u=\left(\begin{array}{ccc}
1 & 1 & 1 \\
0 & 0 & 0 \\
-1 & -1 & -1
\end{array}\right) \star u .
\end{aligned}
$$

Like the Sobel operator, the Prewitt operator can also evaluate the directional derivatives and is used for edges detection. It also uses three rows/columns to compute the derivative of the specific row/column. However, unlike the Sobel operator, the Prewitt operator considers same weights of all three rows/columns. So, details of the specific row are preserved no better than by the Sobel. Another difference of the Sobel from the Prewitt is that in the case of the image has a little noise, the Sobel operator gives higher accuracy for the task of edge detection.

The gradient magnitude can be computed based on directional derivatives:

$$
|\nabla u|=\sqrt{\left(\frac{\partial u}{\partial x_{1}}\right)^{2}+\left(\frac{\partial u}{\partial x_{2}}\right)^{2}}
$$

In all above cases, the second order derivatives can be acquired by applying the derivative operator twice over the image:

$$
\frac{\partial^{2} u}{\partial x_{1}^{2}}=\frac{\partial}{\partial x_{1}}\left(\frac{\partial u}{\partial x_{1}}\right), \frac{\partial^{2} u}{\partial x_{1} \partial x_{2}}=\frac{\partial}{\partial x_{1}}\left(\frac{\partial u}{\partial x_{2}}\right), \frac{\partial^{2} u}{\partial x_{2}^{2}}=\frac{\partial}{\partial x_{2}}\left(\frac{\partial u}{\partial x_{2}}\right) .
$$

\subsection{Blood Vessels Segmentation Methods}

Because there are many small blood vessels that their intensity is similar to the intensity of other parts of images, applying thresholding segmentation directly in this case is ineffective. The goal of blood vessels segmentation by the adaptive principal curvature is like the goal of several similar works based on the matched filter, Gabor filter, multiscale line detection, etc. is that improve the image curvilinear structure so that the blood vessels intensity is higher and clearer than intensity of other parts. In this case, we propose to use the adaptive principal curvature to get better result. The Figure 1 shows an example of applying the adaptive principal curvature, the maximum principal curvature and the gradient magnitude for the retinal fundus image with ID 01 . We convert the colourful image to grayscale image. The derivative operator used in this case is the Sobel operator.

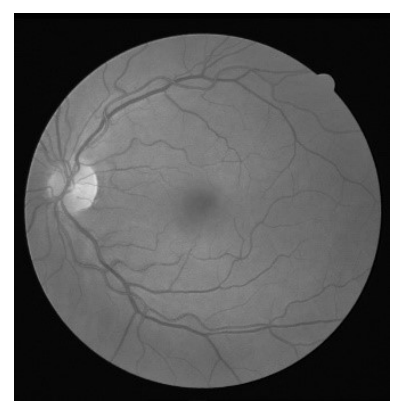

(a) The input retinal fundus image

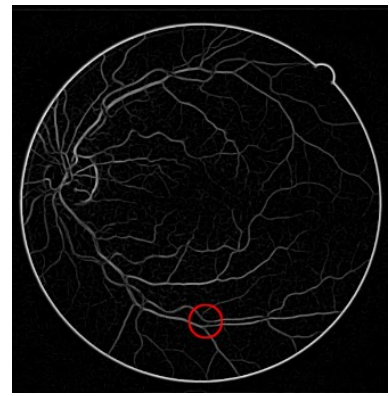

(c) Maximum principal curvature by the Sobel operator

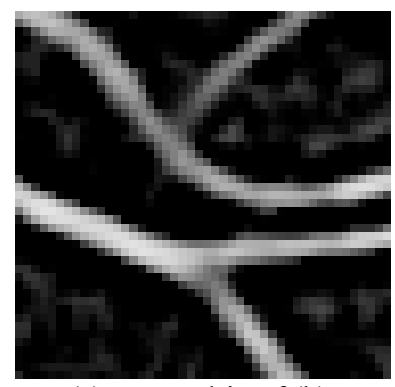

(e) Zoomed-in of (b)

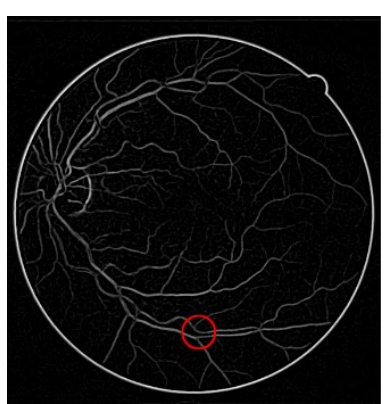

(b) Adaptive principal curvature by the Sobel operator

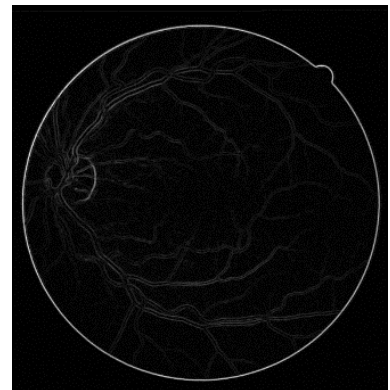

(d) Gradient magnitude by the Sobel operator

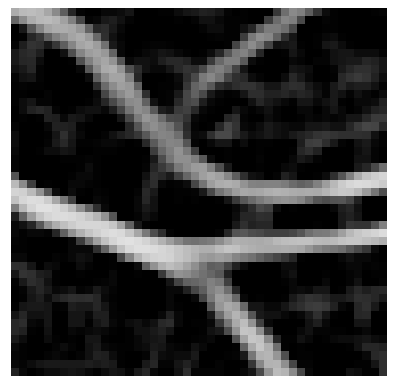

(f) Zoomed-in of (c)
Figure 1. Lines/edges contrast enhancement by applying the adaptive principal curvature, the maximum principal curvature and the gradient magnitude. 
As can be seen, the gradient magnitude improves the contrast of the blood vessels well, but it is still not too clear. The gradient magnitude detects the blood vessels based on the edges and it does not create the continuous structures (sketch structure). By using the principal curvatures (the maximum principal curvature or the adaptive principal curvature), the blood vessels are produced as a clearer structural sketch. The adaptive principal curvature processes better than the maximum principal curvature: the contrast of blood vessels is clearer, and the non-blood-vessels structures are smaller, and are separated from the blood vessels. This can be seen clearly on Figures 1(e) and 1(f) that we zoom in the regions marked by the red colour.

Next step, we need to enhance the contrast of the considered regions again to guarantee that the small blood vessels will not be lost (Prasath, 2017). This task can be done by the contrastlimited adaptive histogram equalization (CLAHE) method (Zuiderveld, 1994) (Yadav, et al., 2014).

\section{Algorithm 1. The blood vessels segmentation methods based on the adaptive principal curvature and derivative operators.}

Input: The image $u$, method to compute derivative.

Output: The segmented image of blood vessels $u_{\text {seg }}$.

\section{Function $u_{\text {seg }} \leftarrow \operatorname{ASeg}(u$, method $)$}

Step 1. Extract the considered region to be segmented.

Step 2. $w \leftarrow$ Apply the Gaussian kernel to the input image $u$.

Step 3. Compute derivatives by the given method:

$$
\begin{gathered}
w_{x_{1}} \leftarrow \frac{\partial w}{\partial x_{1}}, \quad w_{x_{2}} \leftarrow \frac{\partial w}{\partial x_{2}}, \quad w_{x_{1} x_{1}} \leftarrow \frac{\partial}{\partial x_{1}}\left(w_{x_{1}}\right), \\
w_{x_{1} x_{2}} \leftarrow \frac{\partial}{\partial x_{1}}\left(w_{x_{2}}\right), \quad w_{x_{2} x_{2}} \leftarrow \frac{\partial}{\partial x_{2}}\left(w_{x_{2}}\right) .
\end{gathered}
$$

Step 4. $\left\{\lambda_{i j}^{(1)}, \lambda_{i j}^{(2)}\right\} \leftarrow$ Find eigenvalues of the Hessian at every pixel $\boldsymbol{x}$ with position $(i, j)$ in the input image:

$$
\mathbb{H}(\boldsymbol{x}, \sigma) \leftarrow\left(\begin{array}{ll}
w_{x_{1} x_{1}}(\boldsymbol{x}) & w_{x_{1} x_{2}}(\boldsymbol{x}) \\
w_{x_{1} x_{2}}(\boldsymbol{x}) & w_{x_{2} x_{2}}(\boldsymbol{x})
\end{array}\right) .
$$

Step 5. Find the maximum and the minimum principal curvatures:

$$
\begin{array}{ll}
\lambda_{i j}^{+} \leftarrow \max \left\{\lambda_{i j}^{(1)}, \lambda_{i j}^{(2)}\right\}, & \Lambda^{+} \leftarrow\left(\lambda_{i j}^{+}\right)_{m \times n}, \\
\lambda_{i j}^{-} \leftarrow \min \left\{\lambda_{i j}^{(1)}, \lambda_{i j}^{(2)}\right\}, & \Lambda^{-} \leftarrow\left(\lambda_{i j}^{+}\right)_{m \times n} .
\end{array}
$$

Step 6. Compute the adaptive principal curvature:

$$
\Lambda \leftarrow \frac{3 \Lambda^{+}+\Lambda^{-}}{2} \text {. }
$$

Step 7. B $\leftarrow$ Improve limited contrast of the adaptive principal curvatures $\Lambda$ by the CLAHE method.

Step 8. $u_{\text {bin }} \leftarrow$ Segment B by binary threshold segmentation.

Step 9. $u_{\text {seg }} \leftarrow$ Filter out small segments of $u_{\text {bin }}$.

End.
Finally, we must apply the binary thresholding segmentation method to segment the blood vessels based on the acquired result of the adaptive principal curvature. This is helpful to evaluate the Sorensen-Dice and Jaccard metrics and the contour matching score to compare to ground truth. In this case, we use the iterative self-organizing data analysis technique (ISODATA) method (Ridler \& Calvard, 1978) (El-Zaart, 2010). This method is a wellknown $k$-means clustering to define the optimal clusters. For the binary segmentation, this method evaluates $k=2$. So, the global image threshold value will be evaluated based on the image pixels intensities automatically. This value is the threshold used for the binary segmentation (i.e. the segmentation result is only black and white regions). Regions with intensity higher than this threshold are segmented regions (blood vessels). We must notice that, in Algorithm 1, the step 1 is used for extracting the region of interests (ROI). This step is useful to remove the outline border (the border between the black background and the retinal fundus). This step can be completed easily by the binary thresholding segmentation with given thresholding value that is higher than the black colour intensity, for example, with the value 10 . This value is good enough to extract the retinal fundus area from the black background. Otherwise, the step 9 is used for removing small regions by the area opening operation that is a part of the image morphology. This operation removes all connected components (objects) that have fewer than a given number of pixels from the binary image.

The algorithm details of the blood vessels segmentation methods based on the adaptive principal curvature and derivative operators are presented in Algorithm 1.

Corresponding to various derivative evaluation methods, we develop three separated blood vessels segmentation methods. So, we have three blood vessels segmentation methods named ASeg C, ASeg S, ASeg P according to the blood vessel segmentation by the adaptive principal curvature with the central difference, with the Sobel operator, with the Prewitt operator, respectively.

\section{EXPERIMENTAL RESULTS AND DISCUSSIONS}

We handle experiments of the proposed blood vessels segmentation methods based on the adaptive principal curvature and derivative operators in MATLAB 2018a. The configuration of the computing system is Windows 10 Pro with Intel Core i5, 1.6GHz, 4GB $2295 \mathrm{MHz}$ DDR3 RAM memory. The execution time will be smaller if we implement the algorithm on more powerful computing systems or by using parallel processing. We will consider benchmark tests in another future investigation.

\subsection{Synthetic Images}

To handle tests, we implement the proposed blood vessels segmentation methods for DRIVE dataset of the Image Sciences Institute, University Medical Center Utrecht, Netherlands: https://www.isi.uu.nl/Research/Databases/DRIVE/index.html.

In the dataset, there are 20 retinal fundus images. The ground truths are also given. All retinal fundus images are store in TIF format, colourful image and have the same size of $565 \times 584$ pixels. Figure 2 shows all images of DRIVE dataset used for tests. Images IDs are given under their thumbnails (e.g. 01 test.tif - ID is 01, 02_test.tif - ID is $02, \ldots$ ).

In this dataset, there are two collections of ground truth: manual_01 and manual_02. Both are segmented manually by human. In this paper, we only use the manual 01. All ground 
truths are stored in GIF format, binary (black/white) mode, and they have the same ID with corresponding input images.

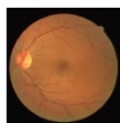

01_test.tif

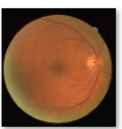

06_test.tif

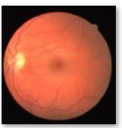

11 test.tif

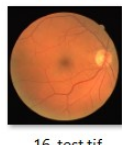

16 test.tif

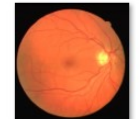

02_test.tif

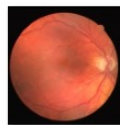

07_test.tif

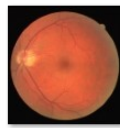

12_test.tif

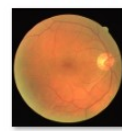

17_test.tif

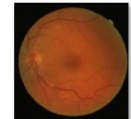

03_test.tif
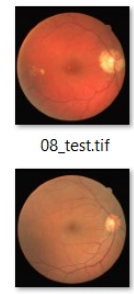

3_test.tif

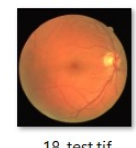

8 test.tif

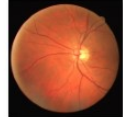

04_test.tif
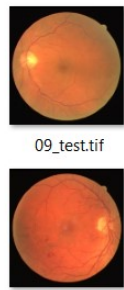

14_test.tif

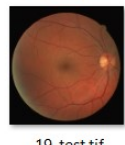

19_test.tif

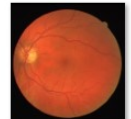

55_test.tif

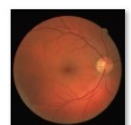

0_test.tif

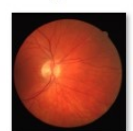

15 test.tif

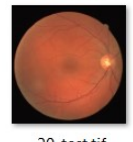

Figure 2. The retinal fundus images for testing

\subsection{Image segmentation quality assessment metrics}

To assess the segmentation quality of the proposed blood vessels segmentation methods, we need the ground truth. We use ground truth manual_01 of DRIVE dataset. In this paper, we consider the following metrics to assess the segmentation quality:

\section{The Sorensen-Dice similarity}

Let consider that $A$ is segmented regions that we need to assess the quality, $B$ is ground truth. The Sorensen-Dice similarity (Abdel \& Allan, 2015) is computed as follows:

$$
\operatorname{dice}(A, B)=\frac{2|A \cap B|}{|A|+|B|},
$$

where | | denotes the set cardinality (number elements of the set). The value of the Sorensen-Dice similarity metric is between 0 and 1 (or $0 \%$ to $100 \%$ ). The higher the Sorensen-Dice value, the better the segmentation result.

\section{The Jaccard similarity}

The Jaccard similarity metric (Abdel \& Allan, 2015) is related to the Sorensen-Dice similarity:

$$
\operatorname{jaccard}(A, B)=\frac{\operatorname{dice}(A, B)}{2-\operatorname{dice}(A, B)} .
$$

The range of the Jaccard value is in $[0,1]$. The higher the Jaccard value, the better the segmentation result.

\section{The contour matching score}

The contour matching score (e.g. BF score) (Gabriela, et al., 2013) measures how close the boundary of segmented regions matches the ground truth boundary. BF score is defined as the harmonic mean of the $\delta$ and $\gamma$ values with the distance error tolerance to decide whether a point on the boundary of segmented regions has a match on the ground truth boundary or not:

$$
B F(A, B)=2 \frac{\delta \gamma}{\gamma+\delta},
$$

where $\delta$ is the ratio of the number of points on the boundary of segmented regions $A$ are closed enough to boundary of the ground truth $B$ to the length of the boundary $A ; \gamma$ is the ratio of the number of points on the boundary of the ground truth $B$ that are closed enough to the boundary of the segmented regions $A$ to the length of the ground truth boundary $B$. The value range of the $\mathrm{BF}$ score is in $[0,1]$. The higher BF score, the better segmentation quality.
We must notice that Sorensen-Dice and Jaccard similarities assess the segmentation quality based on all pixels inside the segmented regions, the $\mathrm{BF}$ score assesses the segmentation quality based on boundaries of the segmented regions and of the ground truth.

\subsection{Test cases}

The first test case: we implement the blood vessels segmentation method based on the adaptive principal curvature with the Sobel operator for the image 01 . In this test, we show results of main steps of the Algorithm 1: the input image (input), the considered region need to be segmented (ROI, step 1), the adaptive principal curvature (step 6), the contrast-limited improvement (CLAHE, step 7), the black-white segmented regions (step 8), the final blood vessels segmentation result (step 9).

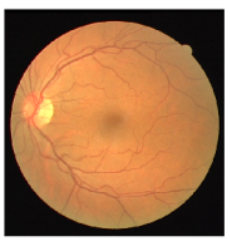

a) Input

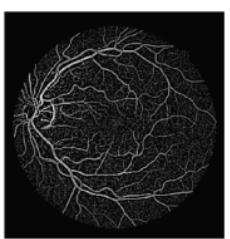

d) CLAHE

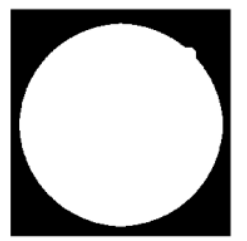

b) $\mathrm{ROI}$

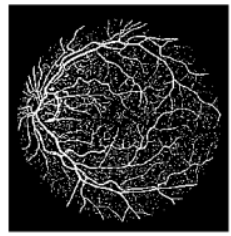

e) Binary segmentation

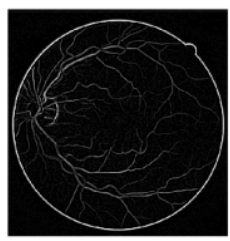

c) The Adaptive principal curvature

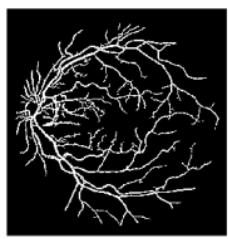

f) Final result
Figure 3. Results by steps of the proposed blood vessel

\begin{tabular}{|c|c|c|c|}
\hline IDs & ASeg_C & ASeg_S & ASeg_P \\
\hline 01 & 0.75197 & 0.76137 & 0.76746 \\
\hline 02 & 0.73862 & 0.76148 & 0.76312 \\
\hline 03 & 0.66144 & 0.69312 & 0.69604 \\
\hline 04 & 0.70939 & 0.73041 & 0.73058 \\
\hline 05 & 0.71797 & 0.73901 & 0.74017 \\
\hline 06 & 0.70606 & 0.71543 & 0.71556 \\
\hline 07 & 0.66939 & 0.70175 & 0.70804 \\
\hline 08 & 0.64035 & 0.65707 & 0.65583 \\
\hline 09 & 0.6967 & 0.72143 & 0.72078 \\
\hline 10 & 0.69791 & 0.69692 & 0.70453 \\
\hline 11 & 0.69515 & 0.70467 & 0.71041 \\
\hline 12 & 0.70061 & 0.72875 & 0.73089 \\
\hline 13 & 0.6935 & 0.71156 & 0.71437 \\
\hline 14 & 0.68939 & 0.71648 & 0.72928 \\
\hline 15 & 0.68723 & 0.66726 & 0.67179 \\
\hline 16 & 0.73092 & 0.76355 & 0.76371 \\
\hline 17 & 0.67768 & 0.71734 & 0.71975 \\
\hline 18 & 0.71179 & 0.75507 & 0.75641 \\
\hline 19 & 0.79706 & 0.81019 & 0.80968 \\
\hline 20 & 0.71732 & 0.75289 & 0.75293 \\
\hline Average & 0.70452 & 0.72529 & $\mathbf{0 . 7 2 8 0 7}$ \\
\hline
\end{tabular}
segmentation method based on the adaptive principal curvature with the Sobel operator.

Table 1 . The Dice similarity value of proposed methods 
The Figure 3 shows the main steps of the proposed blood vessels segmentation method with the Sobel operator. In the first row: the first image from left - the input retinal fundus image, the second one - the considered region, the third one - the adaptive principal curvature. In the second row: the first image from left the improved limited contrast of the adaptive principal curvature, the second one - the binary thresholding segmentation result, the third one - the final blood vessels segmentation result.

\begin{tabular}{|c|c|c|c|}
\hline IDs & ASeg_C & ASeg_S & ASeg_P \\
\hline 01 & 0.60252 & 0.61469 & $\mathbf{0 . 6 2 2 6 6}$ \\
\hline 02 & 0.58557 & 0.61483 & $\mathbf{0 . 6 1 6 9 7}$ \\
\hline 03 & 0.49414 & 0.53036 & $\mathbf{0 . 5 3 3 7 9}$ \\
\hline 04 & 0.54965 & 0.57531 & $\mathbf{0 . 5 7 5 5 3}$ \\
\hline 05 & 0.56003 & 0.58606 & $\mathbf{0 . 5 8 7 5 2}$ \\
\hline 06 & 0.54567 & 0.55694 & $\mathbf{0 . 5 5 7 1}$ \\
\hline 07 & 0.50307 & 0.54054 & $\mathbf{0 . 5 4 8 0 4}$ \\
\hline 08 & 0.47096 & $\mathbf{0 . 4 8 9 2 8}$ & 0.4879 \\
\hline 09 & 0.53456 & $\mathbf{0 . 5 6 4 2 5}$ & 0.56345 \\
\hline 10 & 0.53599 & 0.53482 & $\mathbf{0 . 5 4 3 8 4}$ \\
\hline 11 & 0.53275 & 0.54401 & $\mathbf{0 . 5 5 0 8 8}$ \\
\hline 12 & 0.53919 & 0.57325 & $\mathbf{0 . 5 7 5 9}$ \\
\hline 13 & 0.5308 & 0.55226 & $\mathbf{0 . 5 5 5 6 6}$ \\
\hline 14 & 0.526 & 0.55821 & $\mathbf{0 . 5 7 3 9 1}$ \\
\hline 15 & $\mathbf{0 . 5 2 3 4 9}$ & 0.50067 & 0.50578 \\
\hline 16 & 0.57594 & 0.61753 & $\mathbf{0 . 6 1 7 7 5}$ \\
\hline 17 & 0.51249 & 0.55927 & $\mathbf{0 . 5 6 2 1 9}$ \\
\hline 18 & 0.55254 & 0.60651 & $\mathbf{0 . 6 0 8 2 5}$ \\
\hline 19 & 0.6626 & $\mathbf{0 . 6 8 0 9 5}$ & 0.68022 \\
\hline 20 & 0.55924 & 0.60371 & $\mathbf{0 . 6 0 3 7 5}$ \\
\hline Average & 0.54486 & 0.57017 & $\mathbf{0 . 5 7 3 5 5}$ \\
\hline
\end{tabular}

Table 2 . The Jaccard similarity value of proposed methods

\begin{tabular}{|c|c|c|c|}
\hline IDs & ASeg_C & ASeg_S & ASeg_P \\
\hline 01 & 0.91026 & $\mathbf{0 . 9 4 1 2 1}$ & 0.94038 \\
\hline 02 & 0.89231 & $\mathbf{0 . 9 1 6 4 4}$ & 0.91639 \\
\hline 03 & 0.87607 & $\mathbf{0 . 8 8 7 9 1}$ & 0.8857 \\
\hline 04 & 0.83891 & $\mathbf{0 . 8 6 9 5 8}$ & 0.86831 \\
\hline 05 & 0.8672 & 0.88923 & $\mathbf{0 . 8 8 9 3 9}$ \\
\hline 06 & $\mathbf{0 . 8 7 1 6 8}$ & 0.87008 & 0.86805 \\
\hline 07 & 0.84628 & 0.85591 & $\mathbf{0 . 8 6 2 2 6}$ \\
\hline 08 & 0.82828 & $\mathbf{0 . 8 5 3 5 7}$ & 0.85355 \\
\hline 09 & 0.87153 & 0.8836 & $\mathbf{0 . 8 9 2 5 2}$ \\
\hline 10 & 0.86484 & 0.89213 & $\mathbf{0 . 8 9 7 2}$ \\
\hline 11 & 0.84405 & $\mathbf{0 . 8 6 9 7 7}$ & 0.86465 \\
\hline 12 & 0.88356 & 0.91239 & $\mathbf{0 . 9 1 8 6}$ \\
\hline 13 & 0.85582 & 0.87996 & $\mathbf{0 . 8 8 0 4 6}$ \\
\hline 14 & 0.88204 & 0.91183 & $\mathbf{0 . 9 1 2 8 4}$ \\
\hline 15 & 0.85042 & 0.86602 & $\mathbf{0 . 8 7 0 7 1}$ \\
\hline 16 & 0.88087 & $\mathbf{0 . 8 9 7 5 3}$ & 0.89661 \\
\hline 17 & 0.83117 & 0.85705 & $\mathbf{0 . 8 5 7 7 9}$ \\
\hline 18 & 0.86147 & $\mathbf{0 . 8 9 9 5 1}$ & 0.89815 \\
\hline 19 & 0.9133 & $\mathbf{0 . 9 3 6 6 2}$ & 0.93461 \\
\hline 20 & 0.87758 & $\mathbf{0 . 9 3 0 7 9}$ & 0.93074 \\
\hline Average & 0.86738 & 0.89106 & $\mathbf{0 . 8 9 1 9 5}$ \\
\hline
\end{tabular}

Table 3 . The contour matching score of proposed methods

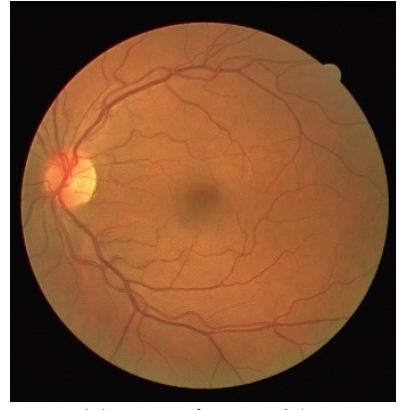

(a) Input image 01

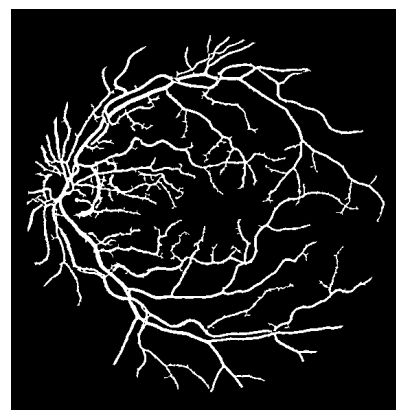

(c) Sobel

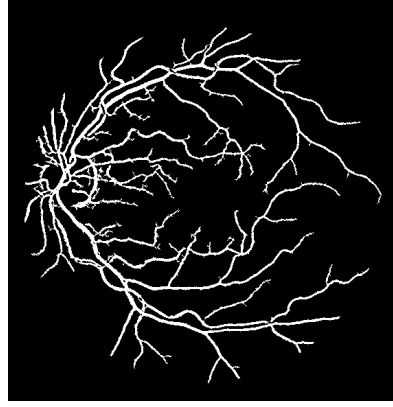

(b) Central difference

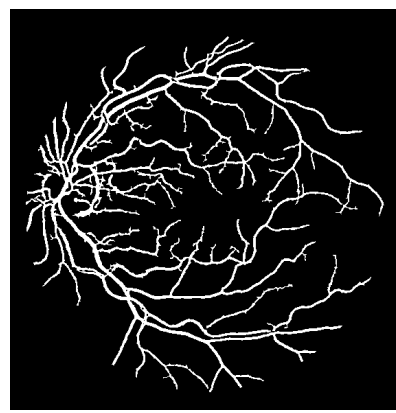

(d) Prewitt
Figure 4. Blood vessels segmentation results for the image 01

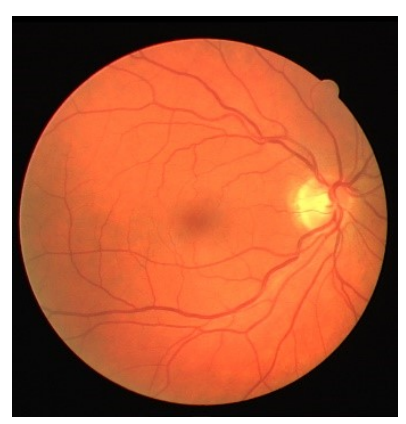

(a) Input image 02

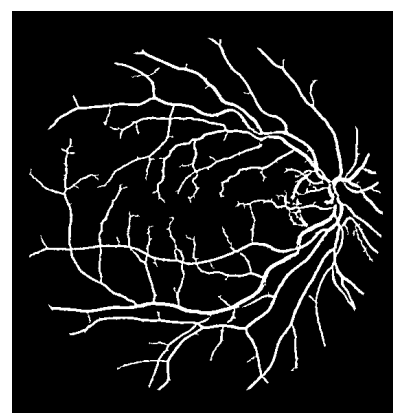

(c) Sobel

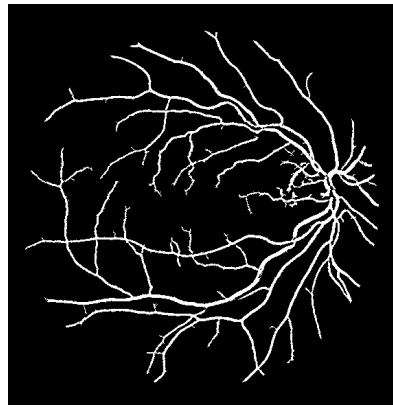

(b) Central difference

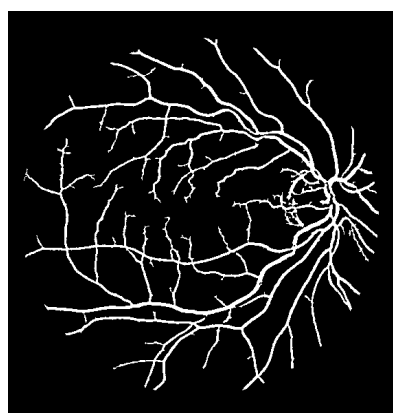

(d) Prewitt
Figure 5. Blood vessels segmentation results for the image 02

By using the adaptive principal curvature, blood vessels are clearly visible. After enhancing the limited contrast, the adaptive principal curvature gets more clearly. Hence, we just need to remove small details to get the final segmentation result.

The second test case: we implement three proposed blood vessels segmentation methods with various derivatives: the central difference, the Sobel and the Prewitt operators on all 20 images of DRIVE dataset. We compare accuracy of three proposed methods based on the Sorensen-Dice similarity, the Jaccard similarity and the contour matching score. 
As can be seen, the blood vessels segmentation quality is very good. All blood vessels are extracted exactly. By the proposed method with the central difference, there are some small details are lost. The result by the proposed methods with the Sobel and the Prewitt operators are better much.

Blood vessels segmentation results are presented in Figures 4-6. As can be seen, the segmentation results by the adaptive principal curvature and the central difference skipped some very small blood vessels. The segmentation results by the principal curvature with the Sobel operator and with the Prewitt operator are better. They can extract very small blood vessels. It is clear on Figures 4-6. In Figure 6, there are small details of blood vessels lost. As a result, there is an unconnected region (the most bottom region). However, this is just a very small region. Besides, we can see that the outline borders of the optic disc (the highest-intensity circle) in the retinal fundus still exist.

The segmentation quality by the Sorensen-Dice error metric is presented in Table 1, the segmentation quality by the Jaccard error metric is presented in Table 2 and for the contour matching score is in Table 3.

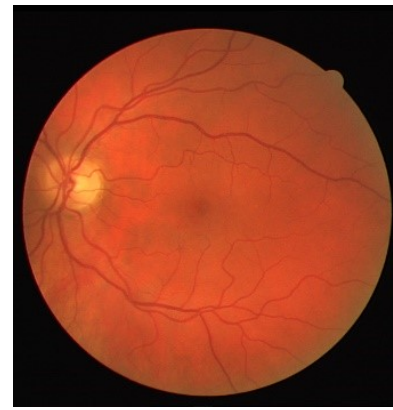

(a) Input image 05

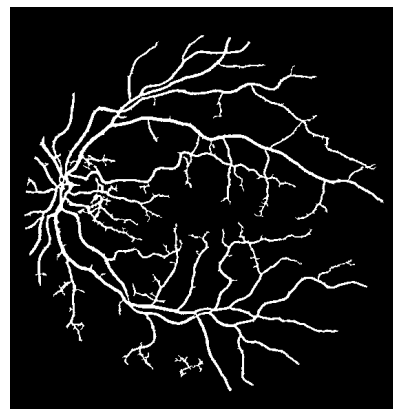

(c) Sobel

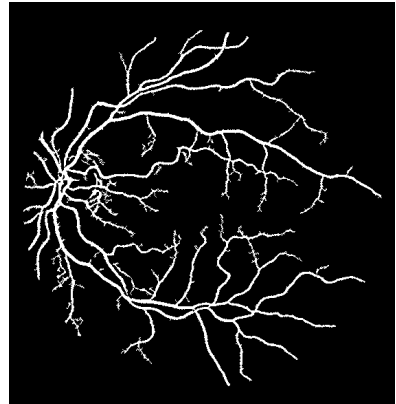

(b) Central difference

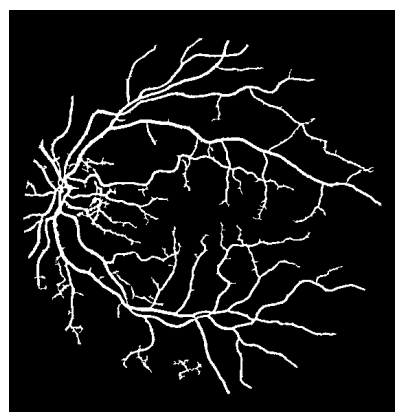

(d) Prewitt
Figure 6. Blood vessels segmentation results for image 05

The Sorensen-Dice error metric and the Jaccard error metric of the segmentation result by the proposed method with the Sobel operator and the Prewitt operator are better than by the central difference. The result by the Prewitt operator is better than the Sobel operator with $17 / 20$ cases and better than the central difference with 19/20 cases. Average values of the SorensenDice metric and the Jaccard metric of the proposed method with the Prewitt operator are highest and followed by the proposed method with the Sobel operator. Lowest average values of the Sorensen-Dice and the Jaccard metrics are by the proposed method with the central difference.

For the contour matching score, the segmentation result by the proposed method with the Sobel operator wins $10 / 20$ cases, with the Prewitt operator wins 9/20 cases and the central difference $1 / 20$ case. However, the average value the contour matching score of the proposed method with the Prewitt operator is still highest. In this case, values of the contour matching score, the Sorensen-Dice metric and the Jaccard metric are quite different because the Sorensen-Dice and the Jaccard metrics assess segmentation results based on whole areas of segmented regions, but the contour matching score only assesses based on the boundary of segmented regions.

\begin{tabular}{|c|c|c|c|c|}
\hline 苞 & : & $\begin{array}{c}\text { Average } \\
\text { Dice score }\end{array}$ & $\begin{array}{c}\text { Average } \\
\text { Jaccard } \\
\text { score }\end{array}$ & $\begin{array}{c}\text { Average BF } \\
\text { score }\end{array}$ \\
\hline \multirow{3}{*}{ 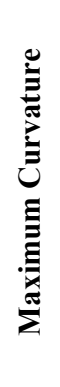 } & 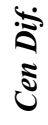 & 0.629183 & 0.462543 & 0.841177 \\
\hline & 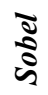 & 0.691565 & 0.530721 & 0.890451 \\
\hline & 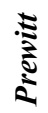 & 0.699151 & 0.539517 & 0.890705 \\
\hline \multirow{3}{*}{ 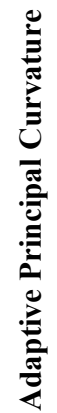 } & $\frac{\dot{5}}{\dot{3}}$ & 0.70452 & 0.54486 & 0.86738 \\
\hline & $\begin{array}{l}\text { ฐँ } \\
\stackrel{5}{0}\end{array}$ & 0.72529 & 0.57017 & 0.89106 \\
\hline & 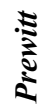 & 0.72807 & 0.57355 & 0.89195 \\
\hline
\end{tabular}

Table 4. Comparison of the average Dice score, the average Jaccard score, the average BF score of the adaptive principal curvature and the maximum principal curvature.

In all cases, the best value of the Sorensen-Dice metric of the proposed method with the central difference is over 0.79 , and lowest value - over 0.64 . For the proposed method with the Sobel operator and with the Prewitt operator are over 0.81 (best) and over 0.65 (lowest); over 0.8 (best) and over 0.65 (lowest), respectively. The best and the lowest values of the Jaccard metric of the central difference are over 0.66 and over 0.47 ; of the Sobel operator - over 0.58 and over 0.48 ; of the Prewitt operator - over 0.68 and over 0.48 , respectively. For the contour matching score, the best and the lowest values of the proposed method with the central difference are over 0.91 (best) and over 0.82 (lowest); with the Sobel operator - over 0.94 (best) and over 0.85 (lowest); with the Prewitt operator - over 0.94 (best) and over 0.85 (lowest), respectively.

Table 4 presents comparison of the average values of the Dice score, of the Jaccard and of the BF score of the adaptive principal curvature and the maximum principal curvature. As can be seen, the proposed methods with the adaptive principal curvature give higher scores than corresponding methods with the maximum principal curvature.

As about the performance, all proposed methods only take less two seconds to complete the blood vessels segmentation task. This is a very good performance and that is suitable to process large images, high-resolution images or video sequences data. 


\section{CONCLUSIONS}

In this paper, we proposed three blood vessels segmentation methods based on the adaptive principal curvature with the central difference, the Sobel operator and the Prewitt operator. All three methods give very good segmentation results on both accuracy and performance. In that, the proposed method based on the adaptive principal curvature with the Prewitt operator has highest accuracy by the Sorensen-Dice and Jaccard metrics.

Our proposed methods can work well with grayscale and colourful images. In the case of colourful images, we can convert images to grayscale mode or process a single colour channel, such as the red, green or blue channel. This does not reduce the method performance for both grayscale or colourful images.

In a future work, we would like to extend the proposed methods to segment blood vessels with higher accuracy by removing the optic disc before applying the proposed segmentation procedure. Moreover, we can also apply multiscale technique (Prasath, et al., 2018) (Prasath, et al., 2015a) (Prasath, et al., 2015b) to improve accuracy; combine with other binary segmentation methods, such as Otsu method, adaptive thresholding method etc. Otherwise, these methods can be also extended to apply to other segmentation problems in medicine (Thanh, et al., 2019, Forthcoming), biomedicine (Thanh \& Dvoenko, 2015a) (Thanh \& Dvoenko, 2015b) (Prasath, 2017), etc.

\section{ACKNOWLEDGEMENTS}

We sincerely thank Dr. B. Van Ginneken and Dr. M. Niemeijer of the Image Sciences Institute, University Medical Center Utrecht, Netherlands by sharing the DRIVE dataset.

\section{REFERENCES}

Abdel, A. T. \& Allan, H., 2015. Metrics for evaluating 3D medical image segmentation: analysis, selection, and tool. $B M C$ Medical Imaging, Volume 15, pp. 1-29.

Aigerim, S. \& Mahmud, H., 2015. Multiscale Blood Vessel Segmentation in Retinal Fundus Images Algorithm Implementation and Analysis. Workshop on Embracing Global Computing in Emerging Economies, Almaty, s.n., pp. 113-121.

El-Zaart, A., 2010. Images thresholding using ISODATA technique with gamma distribution. Pattern Recognition and Image Analysis, 20(1), p. 29-41.

Gabriela, C., Diane, L. \& Florent, P., 2013. What is a good evaluation measure for semantic segmentation. The British Machine Vision Conference, Bristol, s.n., pp. 1-11.

Gonzalez, R. C. \& Woods, R. E., 2008. Digital Image Processing. New Jersey: Pearson Prentice Hall.

Gray, A., 1997. Modern Differential Geometry of Curves and Surfaces with Mathematica. Boca Raton: FL: CRC Press.

Hongli, D. et al., 2007. Principal Curvature-Based Region Detector for Object Recognition. IEEE Conference on Computer Vision and Pattern Recognition, Minneapolis, s.n., pp. 1-8.

Hugo, A.-R.et al., 2018. Blood vessel segmentation in retinal fundus images using Gabor filters, fractional derivatives, and
Expectation Maximization. Applied Mathematics and Computation, 339(5), pp. 568-587.

Moccia, S., Momi, E. D., Hadji, S. E. \& Mattos, L. S., 2018. Blood vessel segmentation algorithms - Review of methods, datasets and evaluation metrics. Computer Methods and Programs in Biomedicine, Volume 158, pp. 71-91.

Ngo, L. \& Han, J.-H., 2017. Advanced deep learning for blood vessel segmentation in retinal fundus images. 5th International Winter Conference on Brain-Computer Interface (BCI), Sabuk, s.n., pp. 91-92.

Patwari, M. et al., 2014. Automated Localization of Optic Disk, Detection of Microaneurysms and Extraction of Blood Vessels to Bypass Angiography. 3rd International Conference on Frontiers of Intelligent Computing: Theory and Applications (FICTA), Bhubaneswar, s.n., pp. 579-587.

Prasath, V. B. S., 2017. Vascularization from Flexible Imaging Color Enhancement (FICE) for polyp localization. Journal of Medicine and Life, 10(2), pp. 147-149.

Prasath, V. B. S. et al., 2015a. Multiscale tensor anisotropic filtering of fluorescence microscopy for denoising microvasculature. IEEE International Symposium on Biomedical Imaging (ISBI), New York, s.n., pp. 540-543.

Prasath, V. B. S., Thanh, D. N. H. \& Hai, N. H., 2018. Regularization Parameter Selection in Image Restoration with Inverse Gradient: Single Scale or Multiscale. IEEE 7th International Conference on Communications and Electronics, Hue, s.n., pp. 278-282.

Prasath, V. B. S. et al., 2015b. Multiscale Tikhonov-Total Variation Image Restoration Using Spatially Varying Edge Coherence Exponent. IEEE Transactions on Image Processing, 24(12), pp. 5220-5235.

Ridler, T. W. \& Calvard, S., 1978. Picture thresholding using an iterative selection method. IEEE Transactions on Systems, Man, and Cybernetics, 8(8), pp. 630-632.

Thanh, D. N. H. \& Dvoenko, S., 2015a. A denoising of biomedical images. The International Archives of Photogrammetry, Remote Sensing and Spatial Information Sciences (Photogrammetric techniques for video surveillance, biometrics and biomedicine), Moscow, s.n., pp. 73-78.

Thanh, D. N. H. \& Dvoenko, S., 2015b. Image noise removal based on Total Variation. Computer Optics, 39(4), pp. 564-571.

Thanh, D. N. H., Prasath, V. B. S. \& Hieu, L. M., 2019, Forthcoming. A Review on CT and X-Ray Images Denoising Methods. Informatica, Volume 43.

Yadav, G., Maheshwari, S. \& Agarwal, A., 2014. Contrast limited adaptive histogram equalization based enhancement for real time video system. International Conference on Advances in Computing, Communications and Informatics (ICACCI), New Delhi, s.n., pp. 2392-2397.

Zuiderveld, K., 1994. Contrast limited adaptive histogram equalization. In: Graphics gems IV. San Diego: Academic Press Professional, pp. 474-485. 